\title{
Click to Fit: Versatile Polyvalent Display on a Peptidomimetic Scaffold
}

\author{
Hangjun Jang, Aaron Fafarman, Justin Holub, Kent Kirshenbaum* \\ Department of Chemistry, New York University, 100 Washington Square East, New York, NY \\ 10003-6688
}

\section{Supporting Information}

\section{A. Peptoid Synthesis.}

Peptoid oligomers were synthesized on Rink Amide resin (Novabiochem, San Diego, CA) using a modification of the submonomer methodology developed by Zuckermann et al ${ }^{1,2}$. The parallel synthesis of peptoid oligomers was fully automated by a robotic workstation (Charybdis Instruments) with software protocols written in-house. All reactions were performed at room temperature unless otherwise specified. The submonomer amines 2-methoxyethylamine and propargylamine were purchased from TCI America; (S)-(+)-1-cyclohexylethylamine was purchased from Lancaster Synthesis, Inc; Benzylamine was obtained from Avocado Research Chemicals Ltd; 3-azido-1aminopropane was synthesized from 3-chloropropylamine and sodium azide as described by Carboni et $\mathrm{al}^{3}$. The fluorophore 6-azido-acetyl-2-(dimethylamino)naphthalene (7) was synthesized by a modification of the method described by Imperiali et al. ${ }^{4}$ Bromoacetic acid and 3'-azido-3'deoxythymidine were purchased from Aldrich. $N, N^{\prime}$ diisopropylcarbodiimide was purchased from Chem-Impex International. Other reagents were obtained from commercial sources and used without additional purification.

Typically, $100 \mathrm{mg}$ Rink amide resin at a loading level of $0.43 \mathrm{mmol} / \mathrm{g}$ was swollen in DMF for 40 minutes before Fmoc deprotection of the resin. Multiple washing steps using DMF were performed between each step described below. Fmoc was cleaved from the resin by $20 \%$ piperidine in DMF, followed by bromoacetylation, in which approximately 20 eq bromoacetic acid (1.2 M in DMF, $8.5 \mathrm{~mL} / \mathrm{g}$ resin) and 24 eq of diisopropylcarbodiimide $(2 \mathrm{~mL} / \mathrm{g}$ resin) were added, followed by $20 \mathrm{~min}$ agitation. Approximately 20 equivalents of a submonomer amine were added according to the preprogrammed sequence. The amine displacement step was effected by 20 min agitation. After several washing steps, the bromoacetylation and the next amine displacement step were repeated until peptoid oligomers with desired sequences were completely synthesized. For analysis, approximately $5 \mathrm{mg}$ of resin was treated with 95\% TFA and $5 \%$ water $(40 \mathrm{~mL} / \mathrm{g}$ resin) to cleave the synthesized peptoid oligomers from the resin over a period of 10 minutes. The cleavage cocktail was evaporated and the peptoid oligomers 
were reconstituted with $1 \mathrm{~mL}$ of $0.1 \%$ TFA in $50 \%$ acetonitrile and $50 \%$ water for HPLC analysis.

Subsequent modification by [3+2] cycloaddition was performed on solid phase support, effecting conjugation at either azide (1-azido-3-aminopropane) or alkyne (propargylamine) side chains.

\section{B. Post-oligomerization Sidechain Modifications by Click Chemistry.}

The resin-bound peptoid was placed into a $1.8 \mathrm{~mL}$ glass vial. Based upon the estimated moles of alkyne or azide functional groups on the scaffold, 7 eq of azide or alkyne coupling partners, respectively, were added. For example, phenylpropargyl ether and 12 were used as partners for conjugation to peptoid azide groups. Benzyl azide, along with compounds 7 and 9 were used as partners for peptoid alkyne groups. To this reaction were added 7 eq of ascorbic acid, 13 eq of $\mathrm{CuI}$ and 17 eq of DIPEA. The organic solvent systems used were either a mixture of 2-butanol/DMF:pyridine 5:3:2, or DMF/pyridine $7: 3$, or 2 -ethoxyethanol/pyridine $7: 3$, as described in scheme $1,2,3$ and 4 . The solvent volume was approximately $0.15 \mathrm{~mL} / \mathrm{mg}$ resin (loading $=0.43 \mathrm{mmol} / \mathrm{g}$ ). After a $15 \mathrm{~min}$ treatment in a bath sonicator to completely dissolve $\mathrm{CuI}$ solid, the reaction mixture became bright greenish-yellow. After tightly capping, the vials were shaken at room temperature. Following overnight reaction, the resin was transferred to a fritted syringe and washed with DMF and DCM several times. The resin was dried under nitrogen gas flow and then cleaved by treatment with $95 \%$ TFA in water for $10 \mathrm{~min}$. The purity of the derivatized peptoid was evaluated by analytical RP-HPLC.

Peptoid oligomers were analyzed by reversed-phase HPLC with a C4 column (Peeke Scientific, Ultra-120, $5 \mu \mathrm{m}, 120 \AA$ A, 2.0 x $50 \mathrm{~mm}$ ) using the Beckman Coulter ${ }^{\mathrm{TM}}$ System Gold $^{\circledR}$ HPLC system and detected by UV absorbance at $214 \mathrm{~nm}$ with a System Gold ${ }^{\circledR} 166$ detector. The data were analyzed with Beckman Coulter 32 Karat $^{\mathrm{TM}}$ software version 5.0. For analytical HPLC, a linear gradient of 5\%-95\% solvent B in solvent A over $30 \mathrm{~min}$ at a flow rate of $0.7 \mathrm{~mL} / \mathrm{min}$ was used. Solvent $\mathrm{A}$ is $0.1 \%$ TFA in HPLC grade water and solvent B is $0.1 \%$ TFA in HPLC grade acetonitrile. Average coupling yields, based upon triplicate reactions, were estimated by reversed-phase analytical HPLC. The $\%$ area of product was divided by the $\%$ area of the reactant to estimate the $\%$ conversion.

To confirm this procedure provides an acceptable estimation of yields, we performed the following comparisons of yield calculated by HPLC analysis and by purified product mass. We synthesized 1-benzyl-4-phenoxyl-1H-1,2,3-triazole by solution phase conjugation of phenyl propargyl ether and benzyl azide and purified it by a simple filtration. Yields determined by weight and estimated by RP-HPLC were in agreement to within $4 \%$. In addition, we synthesized a peptoid octamer and introduced one triazole linkage on it by means of a click chemistry on solid phase. The product was confirmed by MS/MS analysis. The estimated \% yield by RP-HPLC as described above was $72 \%$. The $\%$ yield calculated from the actual yield after Prep HPLC purification of the triazole product and the theoretical yield based upon the resin loading was $71 \%$ (product purity 97\%). These results confirm that, as expected, triazole formation does not 
substantially change the peptoid absorptivity at $214 \mathrm{~nm}$, and validates the application of HPLC chromatograms for yield estimation.

\title{
C. Characterization of $\mathrm{N}$-substituted glycine peptoid oligomers
}

\author{
1. High resolution mass spectrometry of peptoids (LC/MS-TOF)
}

Table S1.

\begin{tabular}{|c|c|c|c|c|c|}
\hline Entry & Charge & Formula & Calc $\mathrm{m} / \mathrm{z}$ & Obs. $\mathrm{m} / \mathrm{z}$ & ppm Error \\
\hline \multirow{2}{*}{ 1a } & +1 & $\mathrm{C}_{72} \mathrm{H}_{109} \mathrm{~N}_{22} \mathrm{O}_{18}$ & 1569.8284 & 1569.8320 & 2.2470 \\
\hline & +2 & $\mathrm{C}_{72} \mathrm{H}_{110} \mathrm{~N}_{22} \mathrm{O}_{18}$ & 785.4178 & 785.4194 & 1.9423 \\
\hline \multirow{2}{*}{$\mathbf{2 a}$} & +1 & $\mathrm{C}_{72} \mathrm{H}_{100} \mathrm{~N}_{13} \mathrm{O}_{18}$ & 1434.7303 & 1434.7334 & 2.1041 \\
\hline & +2 & $\mathrm{C}_{72} \mathrm{H}_{101} \mathrm{~N}_{13} \mathrm{O}_{18}$ & 717.8688 & 717.8701 & 1.7708 \\
\hline \multirow{2}{*}{ 1b } & +2 & $\mathrm{C}_{144} \mathrm{H}_{215} \mathrm{~N}_{43} \mathrm{O}_{36}$ & 1561.3151 & 1561.3148 & -0.2548 \\
\hline & +3 & $\mathrm{C}_{144} \mathrm{H}_{216} \mathrm{~N}_{43} \mathrm{O}_{36}$ & 1041.2125 & 1041.2126 & 0.0408 \\
\hline \multirow{2}{*}{$2 b$} & +2 & $\mathrm{C}_{144} \mathrm{H}_{197} \mathrm{~N}_{25} \mathrm{O}_{36}$ & 1426.2171 & 1426.2223 & 3.6414 \\
\hline & +3 & $\mathrm{C}_{144} \mathrm{H}_{198} \mathrm{~N}_{25} \mathrm{O}_{36}$ & 951.1471 & 951.1508 & 3.8236 \\
\hline \multirow{2}{*}{$4 \mathbf{a}$} & +1 & $\mathrm{C}_{99} \mathrm{H}_{133} \mathrm{~N}_{22} \mathrm{O}_{21}$ & 1966.0010 & 1966.0019 & 0.4489 \\
\hline & +2 & $\mathrm{C}_{99} \mathrm{H}_{134} \mathrm{~N}_{22} \mathrm{O}_{21}$ & 983.5041 & 983.5038 & -0.3527 \\
\hline \multirow{2}{*}{$6 \mathbf{a}$} & +2 & $\mathrm{C}_{93} \mathrm{H}_{122} \mathrm{~N}_{22} \mathrm{O}_{18}$ & 917.4648 & 917.4646 & -0.2449 \\
\hline & +3 & $\mathrm{C}_{93} \mathrm{H}_{123} \mathrm{~N}_{22} \mathrm{O}_{18}$ & 611.9789 & 611.9791 & 0.2037 \\
\hline & +3 & $\mathrm{C}_{198} \mathrm{H}_{264} \mathrm{~N}_{43} \mathrm{O}_{42}$ & 1305.3275 & 1305.3266 & -0.7563 \\
\hline & +4 & $\mathrm{C}_{198} \mathrm{H}_{265} \mathrm{~N}_{43} \mathrm{O}_{42}$ & 979.2475 & 979.2462 & -1.3373 \\
\hline & +3 & $\mathrm{C}_{186} \mathrm{H}_{240} \mathrm{~N}_{43} \mathrm{O}_{36}$ & 1217.2751 & 1217.2775 & 1.9241 \\
\hline & +4 & $\mathrm{C}_{186} \mathrm{H}_{241} \mathrm{~N}_{43} \mathrm{O}_{36}$ & 913.2081 & 913.2090 & 0.8898 \\
\hline \multirow[t]{2}{*}{8} & +2 & $\mathrm{C}_{114} \mathrm{H}_{143} \mathrm{~N}_{25} \mathrm{O}_{21}$ & 1099.0439 & 1099.0409 & -2.7938 \\
\hline & +3 & $\mathrm{C}_{114} \mathrm{H}_{144} \mathrm{~N}_{25} \mathrm{O}_{21}$ & 733.0317 & 733.0301 & -2.2362 \\
\hline \multirow[t]{2}{*}{10} & +2 & $\mathrm{C}_{102} \mathrm{H}_{140} \mathrm{~N}_{28} \mathrm{O}_{30}$ & 1118.5139 & 1118.5106 & -3.0039 \\
\hline & +3 & $\mathrm{C}_{102} \mathrm{H}_{141} \mathrm{~N}_{28} \mathrm{O}_{30}$ & 746.0117 & 746.0093 & -3.2601 \\
\hline & +3 & $\mathrm{C}_{127} \mathrm{H}_{201} \mathrm{~N}_{30} \mathrm{O}_{19}$ & 816.8555 & 816.8537 & -2.3201 \\
\hline & +4 & $\mathrm{C}_{127} \mathrm{H}_{202} \mathrm{~N}_{30} \mathrm{O}_{19}$ & 612.8935 & 612.8913 & -3.6148 \\
\hline
\end{tabular}

For high resolution mass analysis, all the peptoid compounds were analyzed by the Agilent 1100 Series capillary LC system (binary LC pump with degasser, autosampler, thermostatted column compartment, DAD detector) coupled to a time of flight mass spectrometer Agilent 1100 Series LC/MSD TOF. The system was equipped with a dual sprayer electrospray source. The LC/MS analysis involved spraying simultaneously LC effluent through a sample nebulizer and reference compounds using a reference sprayer. Chromatographic conditions involved mobile phase, $100 \%$ water (A) and $100 \%$ acetonitrile (B), containing $0.1 \%$ formic acid. Initial conditions were 95:5 A:B, followed by the gradient to $100 \% \mathrm{~B}$ in $10 \mathrm{~min}$ and wash at 5:95 (A:B) for $5 \mathrm{~min}$. The LC effuent was pumped through 2.1 X $50 \mathrm{~mm}$ Zorbax SB C-8 column at $0.3 \mathrm{~mL} / \mathrm{min}$. Total run time was $15 \mathrm{~min}$. The following MS conditions were used; nebulizer gas $50 \mathrm{psig}$, drying gas $10 \mathrm{l} / \mathrm{min}$, gas temperature $350 \mathrm{C}$, fragmentor $220 \mathrm{~V}$, scan range 100 to 3000 $\mathrm{m} / \mathrm{z}$, transient length 104992 points with 10,000 transients/scan. Exact mass 
measurements were accomplished using reference compounds at $\mathrm{m} / \mathrm{z} 121.050873$ and 922.009798.

\section{Sequencing of Peptoid Oligomers by Ion Trap LC/MS/MS}

All peptoid oligomers in the current study were subjected to $\mathrm{LC}_{\mathrm{MS}}{ }^{2}$ analysis to confirm the linear sequences of the monomer units and to verify site specific side-chain modifications. Herein, we provide representative characterization data. Additional details regarding sequencing will be presented in a separate publication.

All peptoid 12mers and 24mers described in Scheme 2-4, with molecular weights ranging from 1569 to $3912 \mathrm{Da}$, were analyzed by the Agilent 1100 Series capillary LC system coupled to an ion trap mass spectrometer Agilent 1100 Series LC/MSD Trap XCT equipped with an electrospray ion source. All experiments were performed in positive ion mode using auto $\mathrm{MS}^{\mathrm{n}}$ function. All the analyses were performed without any further purification steps after cleaving the compounds from the resin.

LC/MS ${ }^{2}$ using an ion trap of doubly charged parent ion molecules of 12 mer peptoids (m/z 785.6 for compound 1a before modification and m/z 984.3 for compound 4a after modification) resulted in extensive coverage of $\mathrm{Y}$ " and B ions as the most abundant fragment ions (Figure S1 and S2). These findings are consistent with a report of peptoid sequencing using high energy collision-induced dissociation ${ }^{5}$. Fragment ions observed were consistent with anticipated values based on sequences of peptoid starting material and product.

Figure S3 presents MS/MS spectrum of crude 24mer peptoid $\mathbf{6 b}$ following multisite modification by click chemistry. MS/MS on the triply charged ion produced the most structural information in terms of $\mathrm{Y}$ " and B ion coverage in comparison to the information obtained by fragmentation of the doubly or quadruply charged ions (Figure S4 \& Table S2).

Figure S1. Nomenclature of peptoid backbone sequence ions obtained from 1a \& $4 \mathbf{a}$.

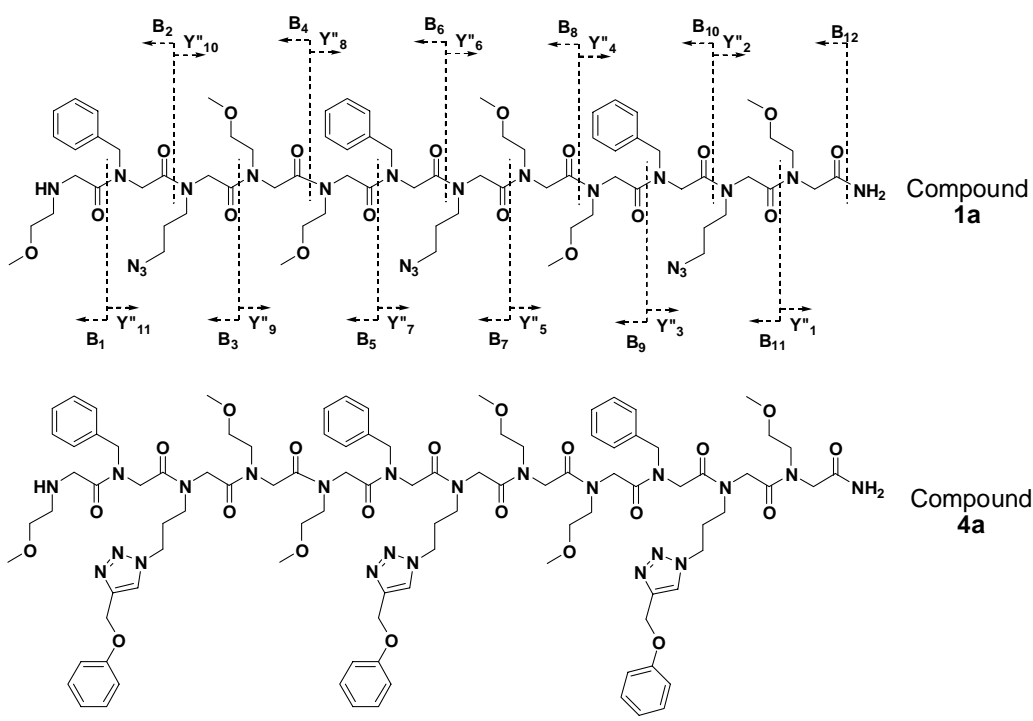


Figure S2. CID mass spectra of $(\mathrm{M}+2 \mathrm{H})^{2+}$ of compound $1 \mathrm{a}$ at $785.6 \mathrm{~m} / \mathrm{z}$ before the click chemistry reaction and $(\mathrm{M}+2 \mathrm{H})^{2+}$ of compound $\mathbf{4 a}$ at $984.3 \mathrm{~m} / \mathrm{z}$ after multisite modification.

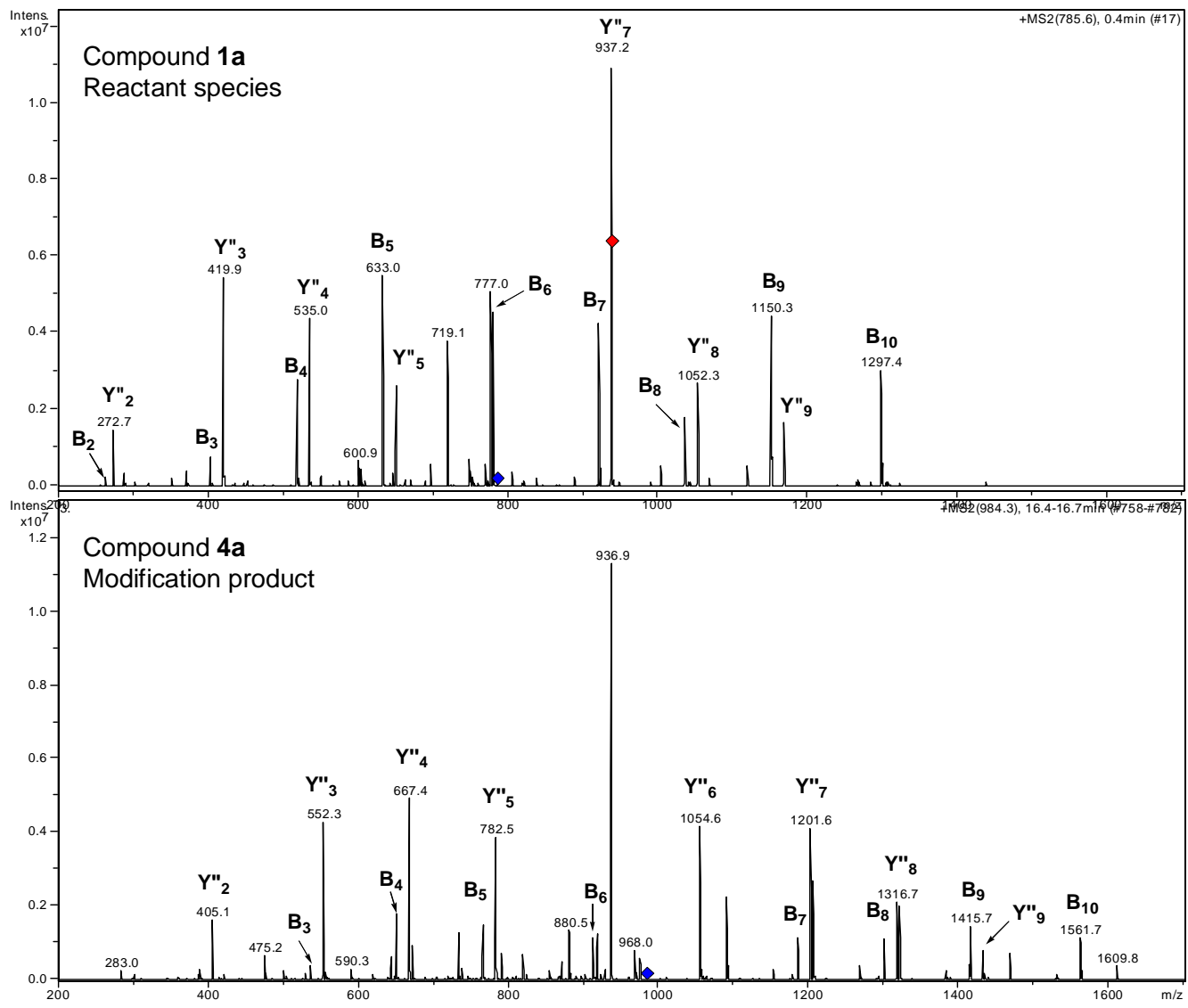

Figure S3. CID mass spectrum of $(\mathrm{M}+3 \mathrm{H})^{3+}$ of compound $\mathbf{6 b}$.

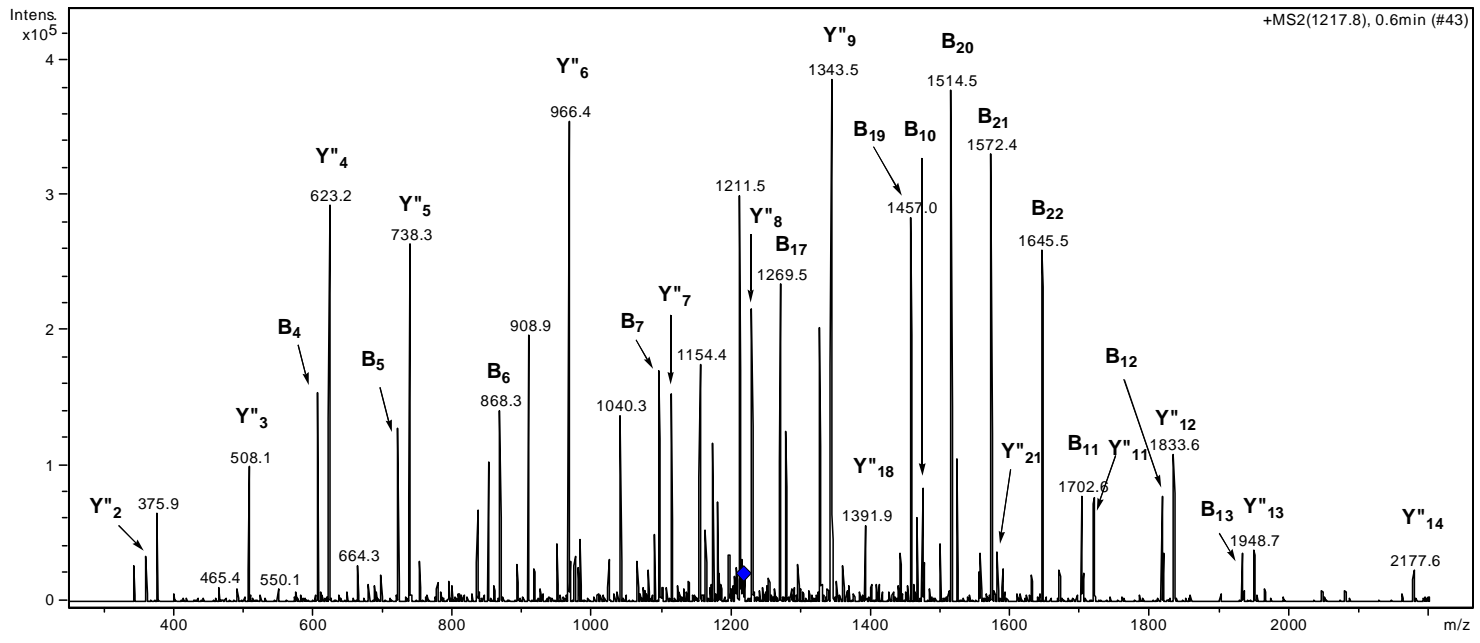


Figure S4. Nomenclature of peptoid backbone sequence ions compounds $\mathbf{6 b}$.

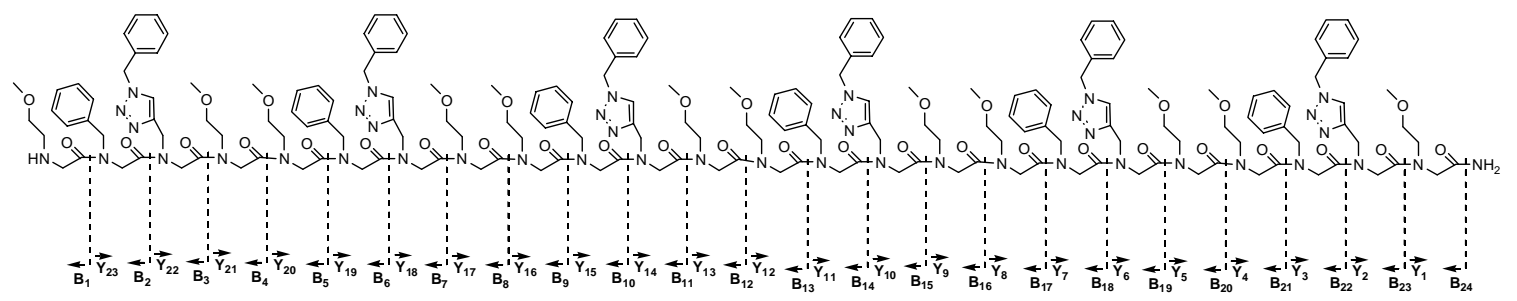

Table S2. B and Y" fragment ions of 24mer peptoid $\mathbf{6 b}$.

\begin{tabular}{|c|c|c|c|c|c|c|c|c|c|}
\hline \multirow{2}{*}{$\begin{array}{c}\text { B } \\
\text { ions }\end{array}$} & \multicolumn{3}{|c|}{ Calc. $\mathrm{m} / \mathrm{z}$ of fragment ions } & \multirow{2}{*}{ Obs $\mathrm{m} / \mathrm{z}$} & \multirow{2}{*}{ Y" ions } & \multicolumn{3}{|c|}{ Calc. $\mathrm{m} / \mathrm{z}$ of fragment ions } & \multirow{2}{*}{ Obs $\mathrm{m} / \mathrm{z}$} \\
\hline & $Z=+1$ & $Z=+2$ & $Z=+3$ & & & $Z=+1$ & $Z=+2$ & $Z=+3$ & \\
\hline $\mathrm{B}_{1}$ & 116.1 & 59.1 & 39.7 & $\mathrm{~N} / \mathrm{A}$ & $Y^{\prime \prime}{ }_{1}$ & 133.1 & 67.1 & 45.7 & $\mathrm{~N} / \mathrm{A}$ \\
\hline $\mathrm{B}_{2}$ & 263.1 & 132.6 & 88.7 & $\mathrm{~N} / \mathrm{A}$ & $\mathrm{Y}_{2}$ & 361.1 & 181.1 & 121.7 & 361.0 \\
\hline $\mathrm{B}_{3}$ & 491.2 & 246.6 & 164.7 & 491.0 & $Y_{3}{ }_{3}$ & 508.3 & 254.7 & 170.8 & 508.1 \\
\hline $\mathrm{B}_{4}$ & 606.3 & 304.2 & 203.1 & 606.2 & $Y_{4}{ }_{4}$ & 623.3 & 312.2 & 209.1 & 623.2 \\
\hline $\mathrm{B}_{5}$ & 721.4 & 361.7 & 241.5 & 721.2 & $\mathrm{Y}_{5}$ & 738.4 & 369.7 & 247.5 & 738.3 \\
\hline $\mathrm{B}_{6}$ & 868.4 & 435.2 & 290.5 & 868.4 & $Y_{6}{ }_{6}$ & 966.5 & 483.8 & 323.5 & 966.4 \\
\hline $\mathrm{B}_{7}$ & 1096.5 & 549.3 & 366.5 & 1096.4 & $Y "{ }_{7}$ & 1113.6 & 557.3 & 372.5 & 1113.4 \\
\hline $\mathrm{B}_{8}$ & 1211.6 & 606.8 & 404.9 & 1211.5 & Y"8 & 1228.6 & 614.8 & 410.9 & 1228.6 \\
\hline $\mathrm{B}_{9}$ & 1326.7 & 664.4 & 443.2 & 1326.4 & Y"9 & 1343.7 & 672.4 & 449.2 & 1343.5 \\
\hline $\mathrm{B}_{10}$ & 1473.7 & 737.9 & 492.2 & 1473.5 & $Y_{10}$ & 1571.8 & 786.4 & 525.3 & $\mathrm{~N} / \mathrm{A}$ \\
\hline $\mathrm{B}_{11}$ & 1701.8 & 851.9 & 568.3 & 1701.6 & $Y^{\prime \prime}{ }_{11}$ & 1718.8 & 859.9 & 574.3 & 1718.6 \\
\hline $\mathrm{B}_{12}$ & 1817.0 & 909.5 & 606.7 & 1816.6 & $Y_{12}$ & 1833.9 & 917.5 & 612.6 & 1833.6 \\
\hline $\mathrm{B}_{13}$ & 1932.0 & 967.0 & 645.0 & 1931.6 & $\mathrm{Y}_{13}$ & 1949.0 & 975.0 & 651.0 & 1948.7 \\
\hline $\mathrm{B}_{14}$ & 2079.0 & 1040.5 & 694.0 & 2078.7 & $Y^{\prime \prime}{ }_{14}$ & 2177.1 & 1089.1 & 727.0 & 2177.6 \\
\hline $\mathrm{B}_{15}$ & 2307.1 & 1154.6 & 770.0 & 1154.4 & $Y^{\prime \prime}{ }_{15}$ & 2324.1 & 1162.6 & 776.0 & 1162.4 \\
\hline $\mathrm{B}_{16}$ & 2422.2 & 1212.1 & 808.4 & $\mathrm{~N} / \mathrm{A}$ & $Y "{ }_{16}$ & 2439.2 & 1220.1 & 814.4 & $\mathrm{~N} / \mathrm{A}$ \\
\hline $\mathrm{B}_{17}$ & 2537.3 & 1269.7 & 846.8 & 1269.5 & $Y "{ }_{17}$ & 2554.3 & 1277.7 & 852.8 & 1277.4 \\
\hline $\mathrm{B}_{18}$ & 2684.3 & 1343.2 & 895.8 & $\mathrm{~N} / \mathrm{A}$ & $Y "{ }_{18}$ & 2782.4 & 1391.7 & 928.8 & 1391.9 \\
\hline $\mathrm{B}_{19}$ & 2912.4 & 1457.2 & 971.8 & 1457.0 & $Y "{ }_{19}$ & 2929.4 & 1465.2 & 977.8 & 1465.5 \\
\hline $\mathrm{B}_{20}$ & 3027.5 & 1514.8 & 1010.2 & 1514.5 & $Y_{20}$ & 3044.5 & 1522.8 & 1016.2 & 1523.0 \\
\hline $\mathrm{B}_{21}$ & 3142.6 & 1572.3 & 1048.5 & 1572.4 & $Y_{21}$ & 3159.7 & 1580.4 & 1054.6 & 1581.0 \\
\hline $\mathrm{B}_{22}$ & 3289.6 & 1645.8 & 1097.5 & 1645.5 & $Y_{22}$ & 3387.7 & 1694.4 & 1130.6 & $\mathrm{~N} / \mathrm{A}$ \\
\hline $\mathrm{B}_{23}$ & 3517.7 & 1759.9 & 1173.6 & $\mathrm{~N} / \mathrm{A}$ & $Y_{23}^{\prime \prime}$ & 3534.7 & 1767.9 & 1179.6 & $\mathrm{~N} / \mathrm{A}$ \\
\hline $\mathrm{B}_{24}$ & 3632.8 & 1817.4 & 1211.9 & $\mathrm{~N} / \mathrm{A}$ & & & & & \\
\hline
\end{tabular}




\section{Sequential Click Chemistry}

We have performed two cycles of post-oligomerization modifications with a unique coupling moiety for each cycle, thus creating further functional diversity on our peptoid scaffolds. Initially, we needed to test the compatibility of heterocyclic triazole linkages on the subsequent peptoid chain elongation. The model sequential click chemistry reactions were effected as described in scheme S1 by our standard procedures (see section B). After the initial click chemistry reaction, the subsequent peptoid chain synthesis showed the expected high coupling efficiency (see Figure S5 compound 15). This finding is noteworthy because of the potential for acylation of sidechain heterocyclic nitrogens ${ }^{6}$. However, our data clearly indicate that subsequent peptoid chain elongation is not affected by triazole linkages formed by click chemistry.

The elongated chain contained one alkyne-functionalized side chain, which was further subjected to a second round of click chemistry reactions with benzyl azide, thus allowing synthesis of a heterogeneous peptoid conjugation product in high yield (Scheme $\mathrm{S} 1$ and Figure S5 compound 16). Therefore, peptoid chain elongation and modification is fully compatible with preformed triazole linkages on the peptoid scaffolds. The feasibility of chain elongation after click chemistry reactions will enable elaboration of chemical and structural diversity through multiple rounds of click chemistry reactions.

Scheme S1. Model reaction for sequential click chemistry

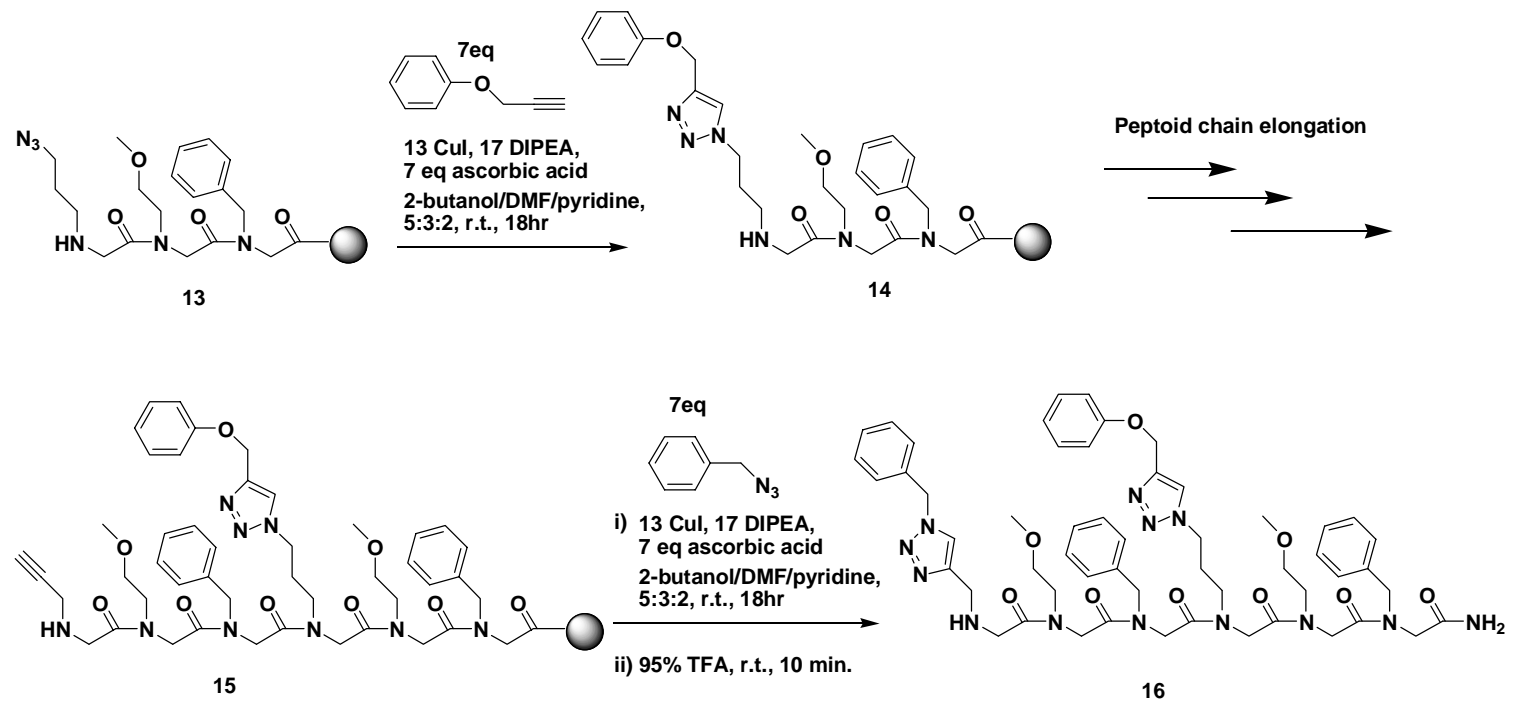


Figure S5. HPLC traces for the model sequential click reaction in Scheme S1

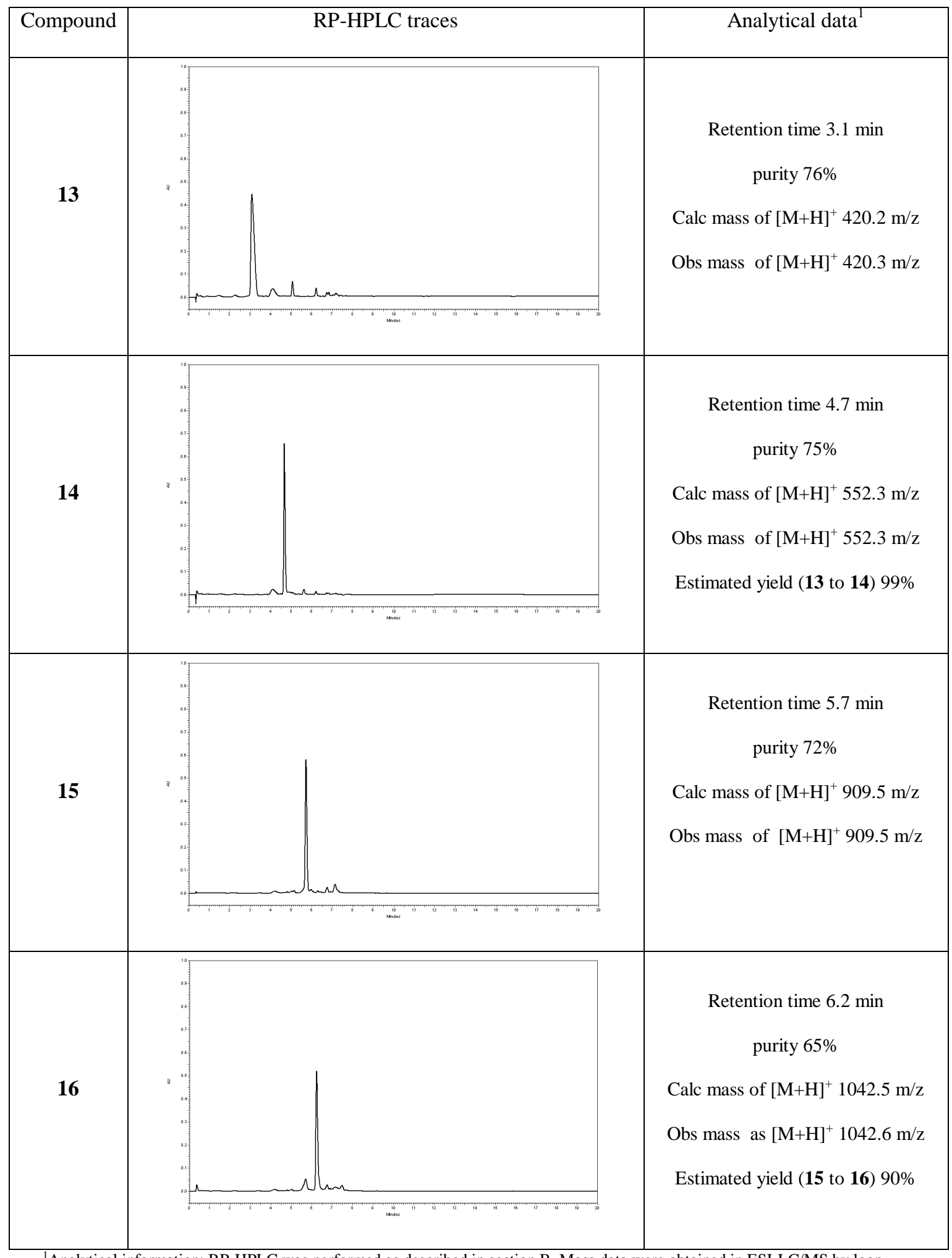

${ }^{1}$ Analytical information: RP-HPLC was performed as described in section B. Mass data were obtained in ESI-LC/MS by loop injection of crude peptoid samples in positive ion mode. 
Reference List

(1) Zuckermann, R. N.; Kerr, J. M.; Kent, S. B. H.; Moos, W. H. J. Am. Chem. Soc. 1992, 114, 10646-10647.

(2) Horn, T.; Lee, B. C.; Dill, K. A.; Zuckermann, R. N. Bioconjug. Chem. 2004, 15, 42835 .

(3) Carboni, B.; Benalil, A.; Vaultier, M. J. Org. Chem. 1993, 58, 3736-3741.

(4) Nitz, M.; Mezo, A. R.; Ali, M. H.; Imperiali, B. Chem. Commun. 2002, 1912-1913

(5) Heerma, W.; Versluis, C.; de Koster, C. G.; Kruijtzer, J. A.; Zigrovic, I.; Liskamp, R. M. Rapid Commun. Mass Spectrom. 1996, 10, 459-464.

(6) Burkoth, T. S.; Fafarman, A. T.; Charych, D. H.; Connolly, M. D.; Zuckermann,R. N. J. Am. Chem. Soc. 2003, 125, 8841-8845 